International Journal of Engineering \& Technology, $7(2.7)(2018) 323-325$
International Journal of Engineering \& Technology
Website: $w w w . s c i e n c e p u b c o . c o m / i n d e x . p h p / I J E T$
Research Paper

\title{
Interactive online book system
}

\author{
P. Bindu Priya ${ }^{1 *}$, G. Rohit ${ }^{1}$, Mrs. P. S. G. Aruna Sri ${ }^{2}$ \\ ${ }^{1}$ B. Tech Student, Department of Electronics \& Computer Engineering KLEF, AP, India \\ ${ }^{2}$ Associate Professor, Department of Electronics \& Computer Engineering, KLEF, AP, India Green Fields, \\ Vaddeswaram, Guntur, Andhra Pradesh 522502 \\ *Corresponding author E-mail: pasumarthipriya498@gmail.com
}

\begin{abstract}
The basic objective of making interactive book system is to make a web application. Which serves individuals with each one of the functionalities gave in a book shop. Few conditions are taken as consideration to developing this application, first one is customer view and second one is administrative view. In customer view customer can upload the book and download a book and they can add the anno-tations to know about the book. They can provide the suggestions about that book. That can be viewed by administrator and admin can solve as per the instructions. The architecture of application is plot both the customer view and administrative view. Administrator has to verify customer identity by this application. This application is processed by JAVA programming language, For designing we are using HTML and CSS.
\end{abstract}

Keywords: Adding the Book; Delete the User; Making Annotations; Suggestions and Comments.

\section{Introduction}

In this site will help its client to peruse the books on the web and play out specific operations that incorporates Annotation, remarks, features and so forth both publicly and secretly. The prerequisite of synchronizing people in general explanation and remarks can influence the general stream of information. Likewise, separating the remarks and keep a check and adjust of the credibility and unwavering quality of the comments refreshed by various clients is additionally an essential and troublesome undertaking to deal with. In this Website is novel of its form. It is independent and doesn't require any incorporation from some other system to work. The real stream of the framework originates all things considered or outside clients who transfer and refresh the book show on the web. Moreover, customers can download the books assume a noteworthy part in the positioning of the book. As contrasted and client point of view, those book are intelligent wellspring of get-ting learning in an uncommon way. Rather than simply perusing the book, the client can exemplify with the information and re-marks of individuals around the globe. To put it plainly, the client can see the book with the viewpoint of various clients who are perusing and can profit different clients with his remarks, explana-tions and input We analyzed the way the customer/client can pro-vide the instructions and how as often as possible those educators begin talks connect with a couple of components - For analysis posting rates, lengths of exchange strings, and understudy study reactions on the instructive experience. We found that the manners by which teachers post to gatherings can impact understudies' gathering exchanges and observations, however not generally in expected ways. Everything considered, visit posting by instructors did not speedy more understudy postings, when the more the edu-cators posted, then the discussions are getting lesser in general[1]. Then again, while most understudies evaluated their instructive experience very, educators who posted every now and again were judged overall to be more eager and master than the individuals who did not. Obviously the quantity of understudy postings and the rate at which educators take an interest are not basic pointers of the nature of gathering dialogs. We have to discover more in-conspicuous measures of the adequacy of non-concurrent ex-change gatherings for learning and instructing.

\section{Related work}

In this examination, we have investigated the way the educator can evaluation of post exchange gatherings in an online applica-tions composed by a constructive-type display. we confined our investigation to a application in which discourse gatherings has been intended to urge understudies to start the majority of discus-sion and they will provide the solution for each and every us-er/customer inquiries, with direction and support from the educa-tor which is suitable to the customer. The achievement and gen-erally of exchange gatherings like these needs to do the nature of the prevalence student/user- understudy associations, in addition to the profundity of realizing which happens inside these discus-sions. These results are difficult to measure However they meas-ure are quantifiable that, however oversimplified, qualification give some sign of what level of teacher mediation[2] makes for a compelling discourse discussion. Seemingly, an important (if not adequate) condition for a discourse discussion to help profound learning is for it to contain a sizable number of postings by under-studies. We likewise propose that a dialog gathering will probably help profound learning in the event that it contains countless dis-course 'strings' (where a string is developed from an underlying posting and the answers revived, shaping a broadened offbeat 'discussion' on a specific theme or issue). In this examination we have estimated the general number of understudy postings in gath-erings, as contrasted and the quantity of postings by educators, in addition such that incessant postings by teachers have on the length of strings.

The normal origination of the online talk gathering the way of a virtual learning condition in which understudies are probably going to gain as much from each other as from course materials or 
addresses. What they realize can be seen not even an item, however as an innovative intellectual procedure of presenting thoughts, having them censured or developed, and having the capacity to reshape thoughts in the light of companion exchanges.[3] So also, it is trusted that by pondering associates commitments in online talks, understudies take part in higher-arrange handling of data, at that point through the demonstration of articulating their own particular developing comprehension, are driven towards the de-velopment of individual significance which isn't individualistic, but instead a result of the understudies' connection.

\section{Frame work}

We proposed the interactive online book system before we are giving the project mechanism we initialize the intuition there on Here we have to modules mainly admin interface and use interface.

a) Admin

In admin interface he can add the book and delete book and delete user and view suggestions. In order to add book he can read the book and admin can also have the permission to read the user book and delete the user book and view the users suggestions with their user name.

b) User

In this user interface user can search book and he can download book and upload book and after study the book user can access the annotations for the book that means when the user read the content of the book he can add the page number and text of the paragraphs to guide the next person understand [3]. He can post the suggestions for the admin.

IBook is an electronic application which has been intended to give the clients the office of perusing or looking through the books on the web, in this way its design conveys every one of the modules of the application. The framework comprise two primary perspectives, one is client and the other is chairman.

Both the perspectives are very much characterized in the design and their capacities are featured in the chart demonstrated as fol-lows. Aside from the perspectives expressed, an information [4] base framework has been proficient with the application which monitors the stream of information on the site. The designing is arranged to such an extent for both customer and administrator need to first authorized themselves on the site before profiting the workplaces gave by the iBook. Web Interface: An online electron-ic application is outlined, with the objective that the client can get to the application easily.

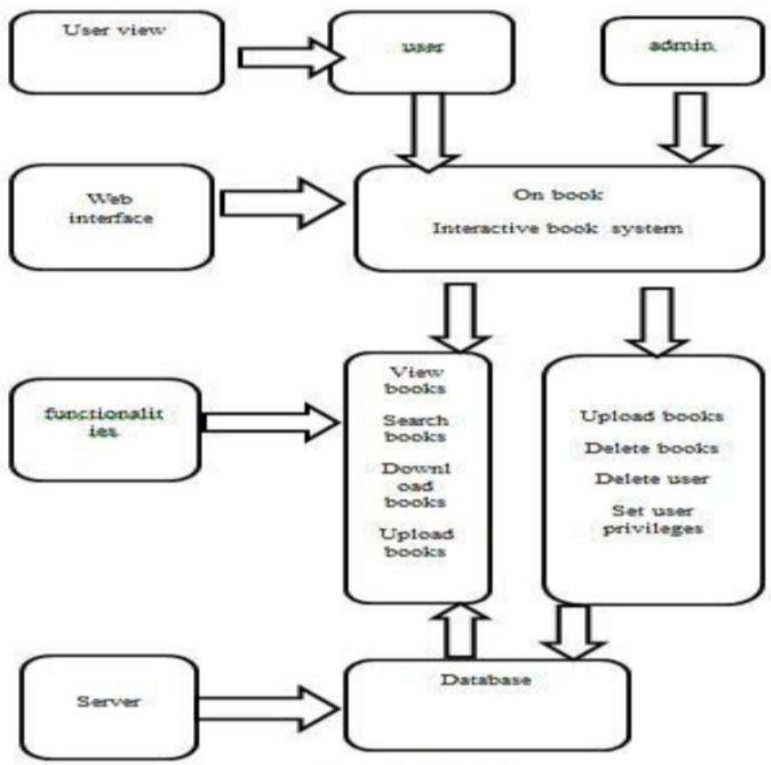

Fig. 1: Architecture

\section{System implementation}

In this application we need to executed the utilization and administrator interface they both have usefulness to uploading the book add comment to comprehend the second client to get the data about aggregate book. The utilization of this application will em-power clients to rate the book too, henceforth the book with all the all the more clear and important information with respect to a particular subject which will be more interested will come into highlight and more people can benefit by it[5]. It is a possibly imaginative plan to bring books identified with comparable classi-fications together so it frames both a library and dialog gathering. They demonstrate the claim by giving the quantity of individuals who have mindfulness and furthermore we can read the book in the website.
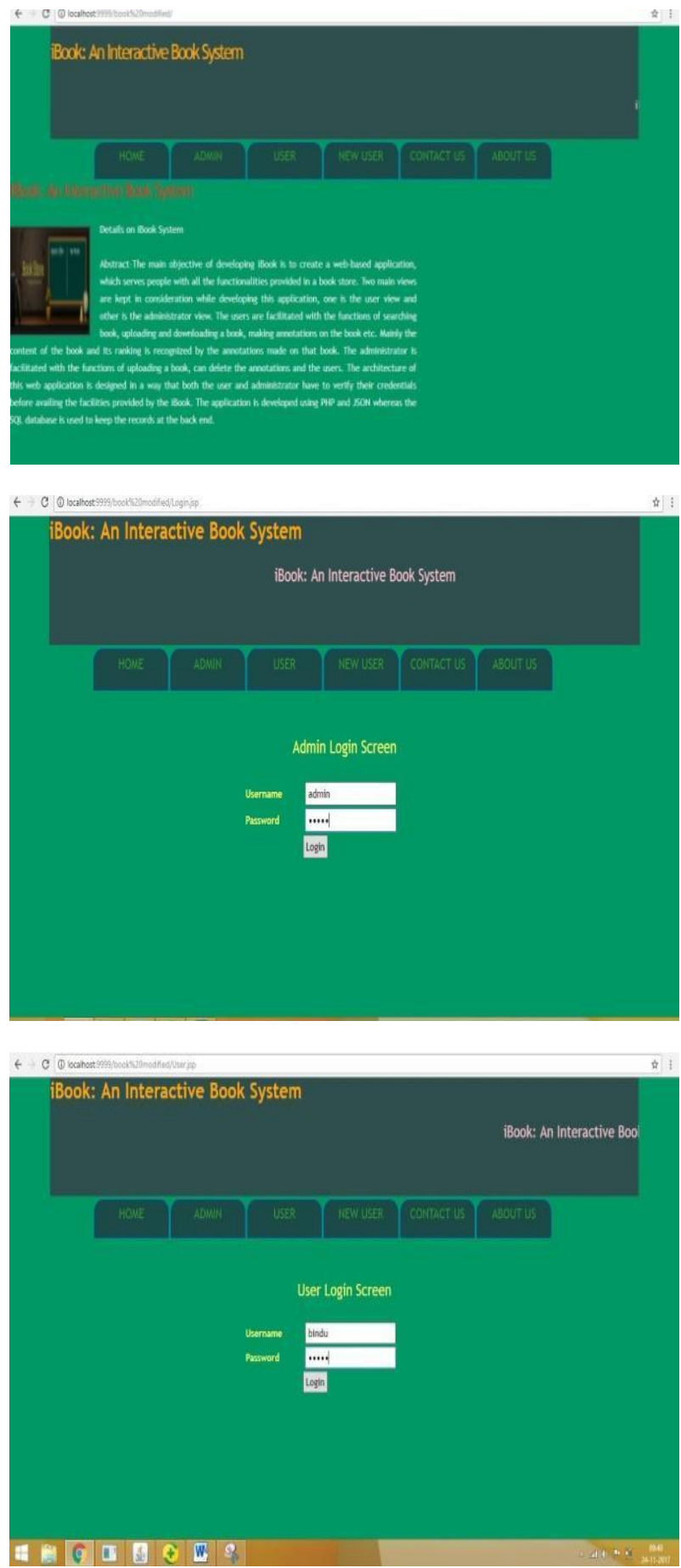


\section{Future work and conclusion}

Interactive Book System is the web application intended for different kinds of clients to profit all the practical offices online gave by a book shop. The current rendition of interactive Book system offers extensive variety of capacities that a client can perform the uploading and download and admin can also upload and delete the data .User can easily find the better solution to read the book online by using annotations and spell checker.

\section{Acknowledgment}

We express our sincere thankfulness to our project guide Mrs. P. S. G. Arunasri for his successful guidance to our project. Without his help, it would be a tough job to accomplish. We thank our guide for his encouragement throughout out period of work.

We also thank our Head of theDepartment (ECSE) Dr. K. Raghava Rao for providing us all the necessary facilities.

\section{References}

[1] G. I. E-book, "Best interactive e-book," 2013. Available: http://www.bestinteractiveebooks.com/about.

[2] S. LaBarre, "Fast Co-design," MIT, 2013. Available:http://www.fastcodesign.com/3026104/awearable- book-thatprograms-you-with-feelings

[3] J. Brownlee, "Fast co-design," Available: http://www.fastcodesign.com/3026104/a-wearable-bookthat- programs-you-with-feelings.

[4] 1. M. J. Pazzani "A Framework for Collaborative Content-Based and Demographic Filtering" <em>Artificial Intelligence Review</em> vol. 13 no. 5 pp. $393-4081999$.

[5] Wright, Scott, and John Street. "Democracy, deliberation and design: the case of online discussion forums. "NewMedia\& Society 9.5 (2007). 